\title{
Modeling and Characterization of Spectrum White Spaces for underlay Cognitive Radio Networks
}

\author{
Vicent Pla, Jose-Ramon Vidal, Jorge Martinez-Bauset and Luis Guijarro \\ Universidad Politécnica de Valencia (UPV), ETSIT Camí de Vera s/n, 46022 Valencia, Spain
}

\begin{abstract}
Cognitive Radio (CR) networks are envisaged as the key technology to realize dynamic spectrum access and solving the scarcity of radio spectrum. Having a temporal characterization of the spectrum white spaces in the primary network is a key element for studying and designing radio resource management mechanisms in CR networks. In that sense, most of the studies in the literature rely on an ON-OFF model with exponentially distributed on and off times. The usage of that model, however, is principally based on its analytical tractability.

In this paper we propose a versatile Markovian model for the duration of the spectrum white spaces. Our model builds on a simple model of the channel holding time (CHT) in the primary network and then matrix-analytic techniques are applied to derive and analyze the duration of the white spaces. Despite its simplicity, the proposed approach is proven to be able to model very accurately scenarios where the CHT distribution is of a more complex type not amenable to mathematical analysis. Our numerical results show that the duration of the white spaces exhibits a low sensitivity to the distribution of the channel holding time beyond the mean.
\end{abstract}

\section{INTRODUCTION}

Cognitive Radio (CR) networks are envisaged as the key technology to realize dynamic spectrum access. Such paradigm shift in wireless communications aims at solving the scarcity of radio spectrum [1]. The CR concept proposes to boost spectrum utilization by allowing CR users (secondary users, $\mathrm{SU})$ to access the licensed wireless channel in an opportunistic manner so that interference to licensed users (primary users, PU) is kept to a minimum.

The idea of CR is undoubtedly compelling and its realization will induce a huge advance in wireless communications. However, there are many challenges and open questions that have to be addressed before CR networks become practically realizable [2]. Most of the existing studies and proposals rely on some model of the spectrum availability resulting from PUs activity. The ON-OFF model with exponentially distributed on and off times is perhaps the most widely adopted model. While that model might be adequate for some studies, in some others it can fail to capture relevant features, which may have an important impact. Hence, some research is necessary to both asses the applicability range of simpler models (e.g. the aforementioned exponential $\mathrm{ON}-\mathrm{OFF}$ ), and develop more versatile models.

Here we consider a primary network (PN) on which the channel holding time (CHT) distribution is known, and aim

This work was supported by the Spanish Government (30\% PGE) and the European Commission (70\% FEDER) through projects TSI2007-66869-C0202 and TIN2008-06739-C04-02/TSI at modeling and studying the statistical properties of the channel idle time. Specifically, we first employ a two-phase Coxian distribution for the CHT in the PN and derive the distribution for the duration of the channel idle time. Secondly, the distribution of the channel idle time when the CHT is lognormally distributed is computed by means of computer simulation, and the results are compared with those obtained analytically. Note that the channel idle time from the PN viewpoint, is the channel available time from the secondary network (SN) perspective. The assumption that the CHT distribution is something known is motivated by the fact that it has been extensively studied during the last two decades (see, for instance, [3]-[8] and references there in). Furthermore, almost all of the references cited above reported the CHT to be of the lognormal type.

The study in [8] has a quite related motivation and objective to ours: characterization and modeling of PU spectrum usage in the cellular band to enable secondary usage of spectrum. However, our target is the channel idle time which has not been addressed there as the study focuses on a cellular network with CDMA air interface. In [9] a statical characterization is derived for the time interval during which there is at least one idle channel among a pool of several channels. This is different from our results as the idle time characterization that is pursued in this paper refers to one channel. Moreover, in [9] PUs are modeled by an ON-OFF process with exponential sojourn times in ON and OFF states, whereas we allow a more general model for the CHT (ON sojourn time). Finally, in [10] a WLAN $802.11 \mathrm{~b}$ is loaded with synthetic traffic and the channel idle time is statistically characterized. While the purpose of this work is quite similar to ours, the scenario is significantly different as it addresses a network with a single channel which is shared by using a contention based MAC. In contrast, here the PN has several channels which are assigned to the PUs during the whole session.

The rest of the paper is structured as follows. In Section II we consider the case where the CHT is distributed according to a two-phase Coxian distribution, and a matrix-analytic model is developed and analyzed. Based on the results obtained in Section II and on extensive computer simulations, Section III presents a series of numerical experiments aimed at studying the statistical properties of the channel idle time and showing the applicability of our modeling approach. Finally, Section IV concludes the paper. 


\section{Model DESCRIPTION AND ANALYSiS}

We consider a PN that works according to a blocked calls cleared model, e.g. a cellular network. The PN has a total of $c$ channels. Each channel can be used to serve exactly one PU. Secondary users can dynamically access the channels that are unused by the PN but must vacate the channel if a PU appears in that particular channel. Upon arrival, a PU request is assigned a channel from the pool of channels that are not currently used by PUs (it may be or may not be used by a SU). For the study carried out in this paper, the way in which the PN selects a channel from its pool of idle channels will have an impact on the results. Here we focus on a random selection scheme, other schemes are left for further research.

We assume that PUs arrive according to a Poisson process of rate $\lambda$. Such assumption has a theoretical support (if the number of users is sufficiently large and they act independently) [11] and has also been empirically confirmed (see, for instance, [8]). The CHT of PUs is assumed to follow a twophase Coxian distribution.

Under the above considerations the PN can be described by a $M / \mathrm{Cox}_{2} / c / c$ model. We focus our attention in one of the $c$ channels, that will be referred to as the observed channel, and study the random variable corresponding to the the time that the channel remains idle (not used by a PU), that we denote by $T_{\text {off }}$. Note that since the assignment scheme is random, it makes no difference which of the $c$ channels is the observed one.

The distribution of $T_{\text {off }}$ can be derived by considering a CTMC model of the $M / \mathrm{Cox}_{2} / c / c$ system (restricted to those states with at least one idle channel: the observed channel) plus an absorbing state that will be visited when an arrival is assigned the observed channel. Then $T_{\text {off }}$ is the time until the CTMC reaches the absorbing state, i.e., $T_{\text {off }}$ follows a phasetype $(P H)$ distribution with representation $P H(\alpha, S)$ [12].

Next, we introduce the parameter representation of the twophase Coxian distribution that models the CHT (referred to as $\mathrm{Cox}_{2}$ hereafter) and then the construction of $\boldsymbol{\alpha}$ and $\boldsymbol{S}$ is described. The vector $\boldsymbol{\alpha}$ contains the initial $(t=0)$ state probabilities for each of the transient states and the matrix $\boldsymbol{S}$ contains the transition rates among the transient states.

\section{A. Coxian Distribution (Cox 2 )}

The family of Coxian distribution is known to be a subfamily of phase-type distribution. Then our $\mathrm{Cox}_{2} r v$ can be defined by specifying its phase-type representation $P H\left(\boldsymbol{\alpha}_{\mathrm{Cox} 2}, \boldsymbol{S}_{\mathrm{Cox} 2}\right)$, where $\boldsymbol{\alpha}_{\mathrm{Cox} 2}=\left[\begin{array}{ll}0 & 1\end{array}\right]$ and

$$
\boldsymbol{S}_{\mathrm{Cox} 2}=\left[\begin{array}{cc}
-\left(\mu_{1}+\mu_{12}\right) & \mu_{12} \\
0 & -\mu_{2}
\end{array}\right]=\left[\begin{array}{cc}
-\gamma_{1} & p \gamma_{1} \\
0 & -\gamma_{2}
\end{array}\right] .
$$

In the expression above two equivalent parameterizations have been introduced. The first one employs the transition rates $\left(\mu_{1}, \mu_{2}, \mu_{12} \geq 0\right)$ among states, whereas the second one employs the total outgoing rate from the transient states $\left(\gamma_{1}, \gamma_{2} \geq 0\right)$ and the probability of the process visiting state 2 after leaving state $1(p)$.

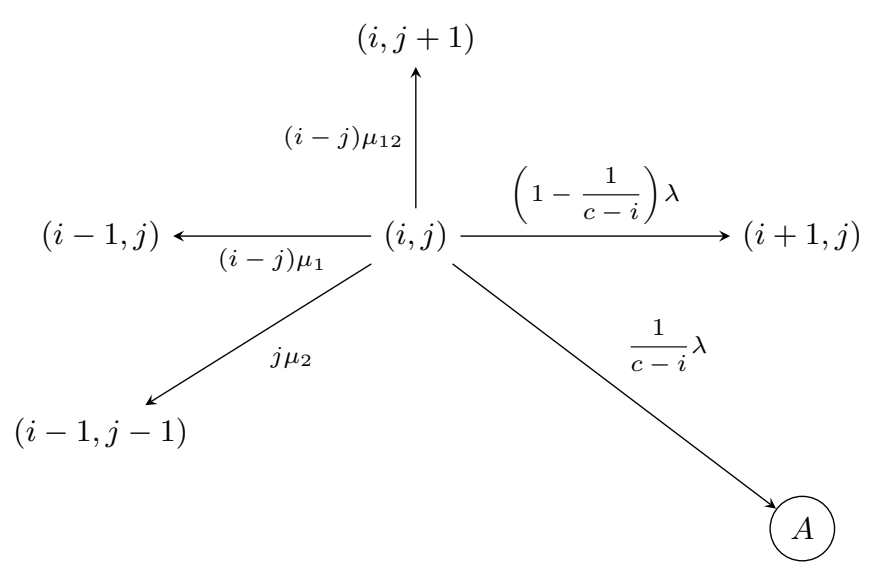

Fig. 1. State transitions.

The average and the variance are then easily found to be

$$
E\left[T_{\text {on }}\right]=\frac{1}{\gamma_{1}}+\frac{p}{\gamma_{2}} \quad \text { and } \quad \sigma^{2}=\frac{1}{\gamma_{1}^{2}}+\frac{p(2-p)}{\gamma_{2}^{2}} .
$$

\section{B. State Space}

Let us denote the state of the system by $(i, j)$ where $i$ represents the number of busy channels (occupied by PUs) and $j$ is the number of these which are at the second stage of their holding time. Then the set of transient states is

$$
\mathcal{S}:=\{(i, j): i=0,1, \ldots, c-1 ; \quad j=0,1, \ldots, i\} .
$$

The number of transient states is $|\mathcal{S}|=\sum_{i=0}^{c-1}(i+1)=c(c+$ $1) / 2$. In the sequel, whenever it is required, we assume that the states in $\mathcal{S}$ are sorted in lexicographical order: $(0,0),(1,0)$, $(1,1), \ldots,(c-1,0),(c-1,1), \ldots,(c-1, c-1)$.

\section{Transition Rates Matrix $\boldsymbol{S}$}

A diagram of the the transition rates is shown in Fig. 1 where the absorbing state is labeled as $A$.

Setting aside the absorbing state we observe that transitions from state $(i, j)$ go to states of the form $(i \pm 1, \cdot)$. Thus, the matrix $\boldsymbol{S}$ has the same structure as the generator $\boldsymbol{Q}$ of a quasi-birth-and-death (QBD) process [12], i.e., it has a blocktridiagonal form

$$
\boldsymbol{S}=\left[\begin{array}{ccccc}
\boldsymbol{A}_{1}^{(0)} & \boldsymbol{A}_{0}^{(0)} & & & \\
\boldsymbol{A}_{2}^{(1)} & \boldsymbol{A}_{1}^{(1)} & \boldsymbol{A}_{0}^{(1)} & & \\
& \ddots & \ddots & \ddots & \\
& & & & \\
& & \boldsymbol{A}_{2}^{(c-2)} & \boldsymbol{A}_{1}^{(c-2)} & \boldsymbol{A}_{0}^{(c-2)} \\
& & & \boldsymbol{A}_{2}^{(c-1)} & \boldsymbol{A}_{1}^{(c-1)}
\end{array}\right] .
$$

The entries in $\boldsymbol{A}_{2}^{(i)}$ are the transition rates between states of the form $(i, \cdot)$ and $(i-1, \cdot)$. In particular the entry $(k, l)$ in $\boldsymbol{A}_{2}^{(i)},\left[\boldsymbol{A}_{2}^{(i)}\right]_{(k, l)}$, is the transition rate from $(i, k)$ to $(i-1, l)$. 
Therefore $\boldsymbol{A}_{2}^{(i)}$ is of size $(i+1) \times i$ and is given by

$$
\boldsymbol{A}_{2}^{(i)}=\mu_{1}\left[\begin{array}{cccc}
i & & & \\
& \ddots & & \\
& & 2 & \\
0 & \cdots & 0 & 0
\end{array}\right]+\mu_{2}\left[\begin{array}{cccc}
0 & 0 & \cdots & 0 \\
1 & & & \\
& 2 & & \\
& & \ddots & \\
& & & i
\end{array}\right] \text {. }
$$

Similarly, $\boldsymbol{A}_{0}^{(i)}$ delineates the transitions rates between states of the form $(i, \cdot)$ and $(i+1, \cdot)$, and $\left[\boldsymbol{A}_{0}^{(i)}\right]_{(k, l)}$ is the transition rate from $(i, k)$ to $(i+1, l)$. Therefore $\boldsymbol{A}_{0}^{(i)}$ is of size $(i+1) \times$ $(i+2)$ and is given by

$$
\boldsymbol{A}_{0}^{(i)}=\left(1-\frac{1}{c-i}\right) \lambda\left[\begin{array}{lllll}
1 & & & & 0 \\
& 1 & & & 0 \\
& & \ddots & & \vdots \\
& & & 1 & 0
\end{array}\right] .
$$

Finally, $\boldsymbol{A}_{1}^{(i)}$ delineates the transitions rates between states of the form $(i, \cdot)$ and $(i, \cdot)$, and $\left[\boldsymbol{A}_{1}^{(i)}\right]_{(k, l)}$ is the transition rate from $(i, k)$ to $(i, l)$ if $k \neq l$, or, if $k=l,-\left[\boldsymbol{A}_{1}^{(i)}\right]_{(k, k)}$ is the total outgoing rate (including that going to the absorbing state) from state $(i, k)$. Therefore $\boldsymbol{A}_{1}^{(i)}$ is a square matrix of size $(i+1) \times(i+1)$ and is given by

$$
\boldsymbol{A}_{1}^{(i)}=\left[\begin{array}{ccccc}
-d_{0} & i \mu_{12} & & & \\
& \ddots & \ddots & & \\
& & -d_{i-2} & 2 \mu_{12} & \\
& & & -d_{i-1} & \mu_{12} \\
& & & & -d_{i}
\end{array}\right]
$$

where $d_{j}=\lambda+i\left(\mu_{1}+\mu_{12}\right)+j\left(\mu_{2}-\mu_{1}-\mu_{12}\right)$.

\section{Initial Probability Vector $\boldsymbol{\alpha}$}

The initial probability of state $(i, j), \pi_{0}(i, j)$, is the probability that at the beginning of a channel idle period the system is at state $(i, j)$ or, equivalently, the probability that upon departure a PU leaves behind the system at state $(i, j)$. Since arrivals and departures occur one at a time, the state probabilities seen by arrivals are the same as those seen by departures (level crossing law [13]) and, since arrivals are Poisson (by virtue of the PASTA property [13]) the latter (and hence also the former) are the same as the stationary probabilities. Therefore the probabilities $\pi_{0}(i, j)$ can be obtained from the stationary probabilities of the $M / \mathrm{Cox}_{2} / c / c$ queue (denoted by $p(i, j)$ ) conditioned to the fact that there is at least one idle channel.

By observing that the $M / \operatorname{Cox}_{2} / c / c$ queue is symmetric (in the sense introduced by Kelly [14]) it follows that: i) the distribution of the number of customers in the queue is insensitive to the distribution of the service time (beyond its mean) and ii) given the number of customers in the queue the amounts of service effort each of the customers have received are independent. Thus we can write

$$
p(i, j)=\frac{a^{i} / i !}{\sum_{k=0}^{c} a^{k} / k !}\left(\begin{array}{l}
i \\
j
\end{array}\right) p_{1}^{i-j} p_{2}^{j},
$$

where $a=\lambda E\left[T_{\mathrm{on}}\right]$ and $p_{l}(l=1,2)$ is the probability of being at stage $l$ in the Markov process induced by the $\mathrm{Cox}_{2}$ distribution. In other words, $p_{1}$ and $p_{2}$ are the stationary probabilities of the CTMC whose generator is given by

$$
\boldsymbol{Q}_{\mathrm{Cox} 2}=\boldsymbol{S}_{\mathrm{Cox} 2}+\left(-\boldsymbol{S}_{\mathrm{Cox} 2} \boldsymbol{e}\right) \boldsymbol{\alpha}_{\mathrm{Cox} 2},
$$

where $e$ is a column vector of ones of the appropriate size.

Upon conditioning on the fact that $i<c$ and substituting the expressions for $p_{1}$ and $p_{2}$ we can write

$$
\pi_{0}(i, j)=\frac{a^{i} / i !}{\sum_{k=0}^{c-1} a^{k} / k !}\left(\begin{array}{l}
i \\
j
\end{array}\right)\left(\frac{\mu_{12}}{\mu_{2}}\right)^{j}\left(1+\frac{\mu_{12}}{\mu_{2}}\right)^{-i} .
$$

Finally, the initial probability vector $\boldsymbol{\alpha}$ can be written as

$\boldsymbol{\alpha}=\left[\begin{array}{lllll}\pi_{0}(0,0) & \cdots & \pi_{0}(c-1,0) & \cdots & \pi_{0}(c-1, c-1)\end{array}\right]$.

\section{E. Analysis}

Phase type distributions is a well studied topic in applied probability [12], [15]. Thus, having specified $T_{\text {off }}$ as a $\mathrm{PH}$ distribution allows us to study its properties by using well established results. Specifically, in the next section, we employ the following results. Recall that the PH representation of $T_{\text {off }}$ is $P H(\boldsymbol{\alpha}, \boldsymbol{S})$ where the construction of $\boldsymbol{\alpha}$ and $\boldsymbol{S}$ has been detailed above.

The distribution function of $T_{\text {off }}$ is given by

$$
F(t)=1-\boldsymbol{\alpha} e^{t \boldsymbol{S}} \boldsymbol{\tau}
$$

where $\tau=-S e$ is a column vector whose entries are the transition rates to the absorbing state. One of the parameters we employ in our numerical study is the 99th percentile $\left(t_{99}\right)$, which can be obtained by solving the equation $\boldsymbol{\alpha} e^{t_{99} S} \boldsymbol{\tau}=$ 0.01 .

The n-th moment is given by $E\left[T_{\text {off }}^{n}\right]=n ! \boldsymbol{\alpha}\left(-\boldsymbol{S}^{-1}\right)^{n} \boldsymbol{e}$. Note, however, that here the derivation of the first moment $E\left[T_{\text {off }}\right]$ can be done in a more simple and general manner by noting that

$$
\frac{E\left[T_{\mathrm{on}}\right]}{E\left[T_{\mathrm{on}}\right]+E\left[T_{\mathrm{off}}\right]}=\frac{a}{c}\left(1-P_{b}(a, c)\right),
$$

where $P_{b}(a, c)$ denotes the blocking probability of a $M / G / c / c$ queue, which is given by the well-known Erlang-B formula. Hence,

$$
E\left[T_{\text {off }}\right]=E\left[T_{\text {on }}\right]\left(\frac{c / a}{1-P_{b}(a, c)}-1\right) .
$$

In order to characterize the tail behavior of $T_{\text {off }}$ we focus on its asymptotic decay rate defined as

$$
\eta=-\lim _{t \rightarrow \infty} \frac{\log P\left(T_{\text {off }}>t\right)}{t},
$$

which is equivalent to $P\left(T_{\text {off }}>t\right)=1-F(t)=K e^{-\eta t}+$ $o\left(e^{-\eta t}\right)$ as $t \rightarrow \infty$, for some $K>0$. Since $T_{\text {off }}$ is a $\mathrm{PH}$ distribution the above limit exists and $-\eta$ is the eigenvalue of $\boldsymbol{S}$ with the largest real part [12]. 


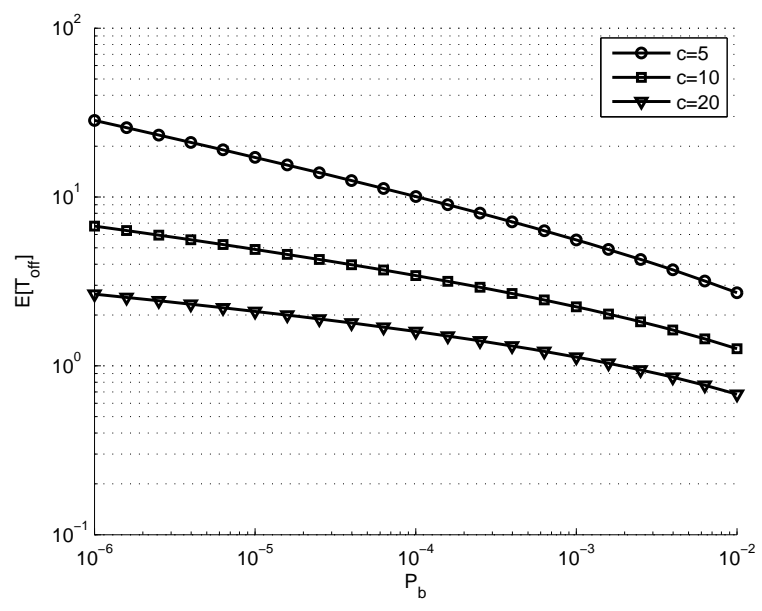

Fig. 2. Mean value.

\section{NUMERICAL STUDY}

Here the analysis of the previous section is applied through a number of numerical experiments in order to study the main characteristics of the channel idle time $\left(T_{\text {off }}\right)$ as a function of several system parameters.

Additionally, a computer simulation based study is carried out for the case in which the CHT follows a lognormal distribution (instead of $\mathrm{Cox}_{2}$ ). The simulation results are compared with those obtained analytically. The main motivation for considering a lognormal-distributed CHT is the fact that there is a significant number of studies ( [3]-[8]) that report a lognormal-based distribution for the CHT. Moreover, the lognormal distribution is qualitatively different from the phasetype distribution, the former is heavy-tailed while the latter has an exponentially decaying tail. Hence, one may think that a lognormal CHT could give rise to a $T_{\text {off }}$ which could not be satisfactorily modeled using an approach that relies on a phase-type CHT, especially with a small number of phases.

In our results all times are normalized with respect to the average CHT, i.e., we set $E\left[T_{\text {on }}\right]=1$. Then we asses the impact of varying the size, load condition and the variability of the CHT. The system size is expressed as the number of channels $(c)$ in the primary network. The load condition is measured in terms of the blocking probability $\left(P_{b}\right)$ for the PUs. We considered a typical worst-case target of $P_{b}=10^{-2}$, so lower values of $P_{b}$ correspond to a load condition outside the busy-hour or to an over-provisioned network. The variability of the CHT is expressed in terms of the coefficient of variation of $T_{\text {on }}, C V\left(T_{\text {on }}\right)=\sigma / E\left[T_{\text {on }}\right]$.

Figure 2 shows the mean value of $T_{\text {on }}$ as a function of the blocking probability for the different systems sizes. It has been computed using the expression in (1) which does not depend on the distribution of $T_{\text {on }}$ beyond its mean. In particular, the variability of the CHT has no impact and the results are exactly the same for a Coxian or a lognormal CHT. As one could expect the mean value of $T_{\text {off }}$ decreases when the load of primary users grows. More specifically that

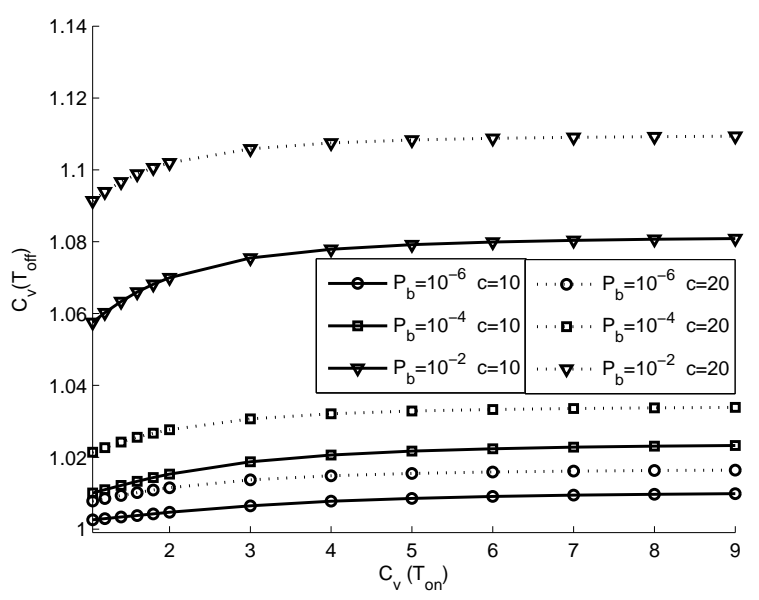

Fig. 3. Coefficient of variation.

trend is roughly linear on a log-log scale. It can also be observed that, for a fixed value of the blocking probability, the value of $E\left[T_{\text {off }}\right]$ decreases for increasing values of the number of channels, since resources are used more efficiently by the primary network as a consequence of the statistical multiplexing effect.

The rest of the characteristics of $T_{\text {off }}$ that we study here are its coefficient of variation, the 99th percentile and the asymptotic decay rate. Unlike the mean value, they are expected to depend on the distribution of $T_{\text {on }}$ beyond its mean. To which extent they do depend on higher moments than $E\left[T_{\text {on }}\right]$ is something that we intend to explore here.

If $T_{\text {on }}$ follows a lognormal distribution, which is defined by two parameters, setting its mean and coefficient of variation fully specifies the distribution. In contrast, the $\operatorname{Cox}_{2}$ depends on three parameters (either $\gamma_{1}, \gamma_{2}, p$ or $\mu_{1}, \mu_{2}, \mu_{12}$ ) and by setting its mean and coefficient of variation $\gamma_{2}$ and $p$ are obtained, while $\gamma_{1}$ can vary freely in the interval $\left(1 / E\left[T_{\text {on }}\right], \infty\right)$.

Figures 3 through 5 plot, respectively, the coefficient of variation, the 99th percentile and the asymptotic decay rate of $T_{\text {off }}$ as a function of the coefficient of variation of $T_{\text {on }}$ for different values of the PN parameters $c$ and $P_{b}$.

Given that we intend to compare the cases where $T_{\text {on }}$ distribution is lognormal and $\mathrm{Cox}_{2}$, a natural way for setting $\gamma_{1}$ would be to choose its value so that, in addition to the mean and coefficient of variation, the 3rd or higher moments of both distributions match. However, a series of numerical experiments (not shown here due to the lack of space) led us to the somewhat surprising conclusion that a constant value for $\gamma_{1}$ yields in general better results than setting it by matching the 3 rd or the 4 th moment. The value of $\gamma_{1}$ has been determined experimentally for each of the parameters of interest. For all the values in Figs. 3 and 4 we used $\gamma_{1}=2.5$, whereas for the values in Fig. $5 \gamma_{1}=1.25$ was used. With those settings, the relative difference between the values obtained using the lognormal distribution and the $\operatorname{Cox}_{2}$ was bounded by $1 \%$ for the values of $C_{v}$ (Fig. 3) and $t_{99}$ 


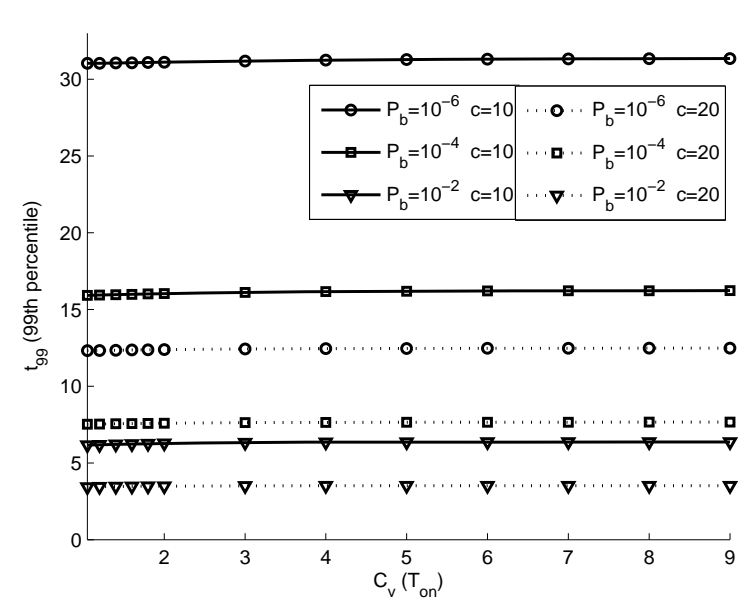

Fig. 4. 99th percentile.

(Fig. 4) and by $7 \%$ for the values of $\eta$ (Fig. 5). For the sake of clarity, Figs. 3 and 4 only plot the set of curves corresponding to the $\mathrm{Cox}_{2}$ distribution. In Fig. 5 the curves for the lognormal distribution are also plotted.

In Fig. 3 we observe that in general the variability of $T_{\text {off }}$ exhibits itself little variation and rather low values $(1<$ $C_{v}\left(T_{\text {off }}\right)<1.11$, even though the value of $C_{v}\left(T_{\text {on }}\right)$ was increased up to 9 . Indeed, for values of $C_{v}\left(T_{\text {on }}\right)$ above 6 the variation of each curve is hardly noticeable. Different curves show that $C_{v}\left(T_{\text {off }}\right)$ increases when $c$ or $P_{b}$ increase, although, as noted, differences are small.

Figure 4 reveals that $t_{99}$ is approximately insensitive to $C_{v}\left(T_{\text {on }}\right)$. Again, a larger (higher $c$ ) or more loaded (higher $P_{b}$ ) PN yields worse results from the SN perspective (lower $t_{99}$ ).

The asymptotic decay rate depicted in Fig. 5. It can be also observed here that the variability of $T_{\text {on }}$ has a low impact on the results.

\section{CONCLUSiON}

In this paper we have proposed a versatile Markovian model for the duration of the spectrum white spaces. Our model builds on a two-phase Coxian distribution for the channel holding time in the primary network and then matrix-analytic techniques are applied to derive and analyze the duration of the white spaces. It is shown that the suggested model yields highly accurate results when the channel holding time distribution is of the lognormal type as has been reported in the literature.

The numerical analysis has shown that the duration of the white spaces exhibits a low sensitivity to the distribution of the channel holding time beyond the mean. In contrast, the size and the load of the primary network have an significant impact. Larger or more loaded primary networks result in worse characteristics from the perspective of the secondary users.

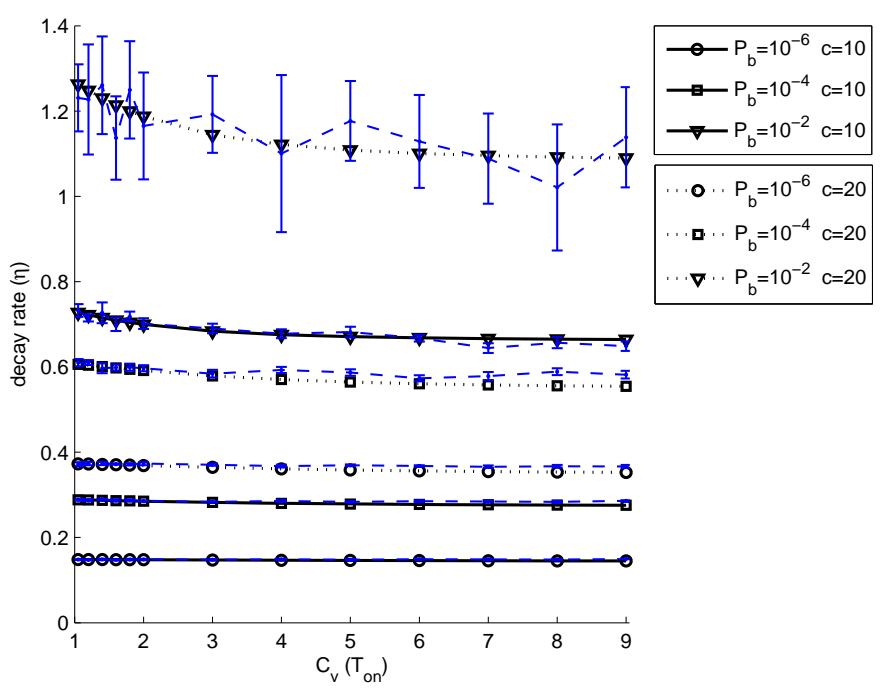

Fig. 5. Asymptotic decay rate. Dashed curves: simulation results for lognormal disbuted $T_{\text {on }}$; vertical bars represent the $95 \%$ confidence intervals.

\section{REFERENCES}

[1] I. Akyildiz, W. Lee, M. Vuran, and S. Mohanty, "NeXt generation/dynamic spectrum access/cognitive radio wireless networks: a survey," Computer Networks, vol. 50, no. 13, pp. 2127-2159, 2006.

[2] I. F. Akyildiz, W. Lee, M. Vuran, and S. Mohanty, "A survey on spectrum management in cognitive radio networks," IEEE Communications Magazine, vol. 46, no. 4, pp. 40-48, 2008

[3] C. Jedrzycki and V. Leung, "Probability distributions of channel holding time in cellular telephony systems," in Proceedings of VTC'96, May 1996, pp. 247-251.

[4] F. Barceló and J. Jordán, "Channel holding time distribution in public telephony systems (PAMR and PCS)," IEEE Transactions on Vehicular Technology, vol. 49, no. 5, pp. 1615-1625, Sep. 2000.

[5] A. Pattavina and A. Parini, "Modelling voice call interarrival and holding time distributions in mobile network," in Proceedings of the 19th International Teletraffic Congress (ITC '05), 2005.

[6] W. Chen, H. Hung, and Y. Lin, "Modeling VoIP Call Holding Times for Telecommunications," IEEE Network, vol. 21, no. 6, pp. 22-28, 2007.

[7] E. Yavuz and V. Leung, "Modeling channel occupancy times for voice traffic in cellular networks," in ICC '07, 2007, pp. 332-337.

[8] D. Willkomm, S. Machiraju, J. Bolot, and A. Wolisz, "Primary user behavior in cellular networks and implications for dynamic spectrum access," IEEE Communications Magazine, vol. 47, no. 3, pp. 88-95, Mar. 2009.

[9] P. K. Tang, Y. H. Chew, and L. C. Ong, "On the distribution of opportunity time for the secondary usage of spectrum," Vehicular Technology, IEEE Transactions on, vol. 58, no. 3, pp. 1517-1527, Mar. 2009.

[10] S. Geirhofer, L. Tong, and B. Sadler, "Dynamic spectrum access in the time domain: Modeling and exploiting white space," IEEE Communications Magazine, vol. 45, no. 5, pp. 66-72, May 2007.

[11] T. Bonald and J. W. Roberts, "Congestion at flow level and the impact of user behaviour," Computer Networks, vol. 42, pp. 521-536, 2003.

[12] M. Neuts, Matrix-geometric Solutions in Stochastic Models: An Algorithmic Approach. The Johns Hopkins University Press, 1981.

[13] R. Nelson, Probability, Stochastic Processes and Queueing Theory. Springer-Verlag, 1995.

[14] F. P. Kelly, Reversibility and Stochastic Networks. Wiley New York, 1979.

[15] G. Latouche and V. Ramaswami, Introduction to Matrix Analytic Methods in Stochastic Modeling. ASA-SIAM, 1999. 